\title{
ON SOME TRIGONOMETRIC POWER SUMS
}

\section{HONGWEI CHEN}

Received 17 June 2001

Using the generating function method, the closed formulas for various power sums of trigonometric functions are established. The computer algebra system Maple is used to carry out the complex calculations.

2000 Mathematics Subject Classification: 33B10.

In [1], we established the trigonometric identity

$$
\sum_{k=0}^{n-1} \frac{1}{1-2 x \cos (2 k \pi / n)+x^{2}}=\frac{n\left(1+x^{n}\right)}{\left(1-x^{n}\right)\left(1-x^{2}\right)},
$$

and reproduced the following elegant formulas [2, 4]:

$$
\begin{aligned}
& \sum_{k=1}^{n-1} \csc ^{2}\left(\frac{k \pi}{n}\right)=\frac{(n-1)(n+1)}{3}, \\
& \sum_{k=1}^{n-1} \tan ^{2}\left(\frac{k \pi}{n}\right)=n(n-1), \quad \text { if } n \text { is odd. }
\end{aligned}
$$

In contrast to Fourier series, these finite power sums are over the angles equally dividing the upper-half plane. Moreover, these beautiful and somewhat surprising sums often arise in analysis. In this note, we extend the above results to the power sums as shown in identities (17), (19), (25), (26), (32), (33), (34), (35), and (36) and in the appendix. The method is based on the generating functions.

To begin, we establish two auxiliary trigonometric identities, derived from the Chebyshev polynomial of the second kind.

Let $U_{n}(x)$ be the Chebyshev polynomial of the second kind [3, pages 7-10]

$$
\begin{aligned}
U_{n}(x) & =\frac{1}{2 i \sqrt{1-x^{2}}}\left[\left(x+i \sqrt{1-x^{2}}\right)^{n+1}-\left(x-i \sqrt{1-x^{2}}\right)^{n+1}\right] \\
& =\sum_{k=0}^{[n / 2]}(-1)^{k}\left(\begin{array}{c}
n-k \\
k
\end{array}\right)(2 x)^{n-2 k} .
\end{aligned}
$$

Setting $x=\cos \theta$ and appealing to De Moivre's theorem, we deduce that

$$
U_{n}(\cos \theta)=\frac{\sin (n+1) \theta}{\sin \theta} .
$$


Thus, $U_{n-1}(x)$ has $n-1$ distinct roots $\cos (k \pi / n), k=1,2, \ldots, n-1$. Since $U_{n-1}(x)$ has leading coefficient $2^{n-1}$, by factorization we have

$$
\prod_{k=1}^{n-1}\left(\cos \theta-\cos \left(\frac{k \pi}{n}\right)\right)=2^{n-1} U_{n-1}(\cos \theta)=2^{n-1} \frac{\sin n \theta}{\sin \theta}
$$

Differentiating (5) logarithmically, we have

$$
\sum_{k=1}^{n-1} \frac{\sin \theta}{\cos \theta-\cos (k \pi / n)}=-n \cot n \theta+\cot \theta .
$$

Replacing $\theta$ by $\pi-\theta$, we find

$$
\sum_{k=1}^{n-1} \frac{\sin \theta}{\cos \theta+\cos (k \pi / n)}=-n \cot n \theta+\cot \theta .
$$

Adding (6) and (7), we obtain

$$
\sum_{k=1}^{n-1} \frac{\sin \theta \cos \theta}{\cos ^{2} \theta-\cos ^{2}(k \pi / n)}=-n \cot n \theta+\cot \theta
$$

or

$$
\sum_{k=0}^{n-1} \frac{\cos \theta}{\cos ^{2} \theta-\cos ^{2}(k \pi / n)}=-n \cot n \theta \csc \theta .
$$

Finally, using the trigonometric identity $\cos ^{2} x=1-\sin ^{2} x$ in (9), we get

$$
\sum_{k=0}^{n-1} \frac{\cos \theta}{\sin ^{2}(k \pi / n)-\sin ^{2} \theta}=-n \cot n \theta \csc \theta .
$$

We now show how to use identities (9) and (10) to establish the closed formulas for various power sums. We start with the evaluation of the power sums for the secant function. Let $p$ be a positive integer. Set

$$
S_{p}(n)=\sum_{k=0}^{n-1} \sec ^{2 p}\left(\frac{k \pi}{n}\right) .
$$

We separate the derivation into two cases.

CASE 1. Let $n$ be an odd positive integer. In order to construct a generating function for $S_{p}(n)$, which has the form

$$
G(n, t)=\sum_{p=1}^{\infty} S_{p}(n) t^{2 p}
$$

we rewrite (9) as

$$
n \cot n \theta \cot \theta=\sum_{k=0}^{n-1} \frac{-\cos ^{2} \theta}{\cos ^{2} \theta-\cos ^{2}(k \pi / n)} .
$$


By expanding the summand as a geometric series, we have

$$
n \cot n \theta \cot \theta=\sum_{k=0}^{n-1} \frac{[\cos \theta / \cos (k \pi / n)]^{2}}{1-[\cos \theta / \cos (k \pi / n)]^{2}}=\sum_{p=1}^{\infty}\left(\sum_{k=0}^{n-1} \sec ^{2 p}\left(\frac{k \pi}{n}\right)\right) \cos ^{2 p} \theta,
$$

where the last summation order has been changed. Thus, comparing (14) to (12) with $t=\cos \theta$, we find the generating function for $S_{p}(n)$

$$
G(n, t)=n \cot n \theta \cot \theta=\frac{n t}{\sqrt{1-t^{2}}} \cot (n \arccos t)=\frac{n t}{\sqrt{1-t^{2}}} \tan (n \arcsin t),
$$

where we have used the fact that $n$ is odd and the relation $\arccos \theta=\pi / 2-\arcsin \theta$. Therefore,

$$
S_{p}(n)=\sum_{k=1}^{n-1} \sec ^{2 p}\left(\frac{k \pi}{n}\right)=\frac{G^{(2 p)}(n, 0)}{(2 p) !},
$$

where $G^{(2 p)}$ indicates the $2 p$ th derivative of $G$ with respect to $t$.

For $p=1$, performing the indicated derivative directly, we find

$$
\sum_{k=0}^{n-1} \sec ^{2}\left(\frac{k \pi}{n}\right)=\frac{G^{\prime \prime}(n, 0)}{2 !}=n^{2}
$$

However, for $p \geq 2$, the expansion of $G^{(2 p)}(n, t)$ is very complicated and inconvenient for the calculation. So, we now demonstrate a derivation carried out with the help of the computer algebra system Maple rather than one done by hand. For example, in Maple, identity (17) is reproduced with the following commands:

$>\operatorname{assume}(n$, integer $)$ :

$>G:=t->n * t * \tan (n * \arcsin (t)) / \operatorname{sart}\left(1-t^{2}\right)$ :

$>\operatorname{subs}(t=0, \operatorname{diff}(G(t), t \$ 2))$ :

$>\operatorname{Sum}\left(\sec ^{2}(k * P i / n), k=0 . . n-1\right)=\operatorname{simplify}(\% / 2 !)$;

$$
\sum_{k=0}^{n-1} \sec ^{2}\left(\frac{k \pi}{n}\right)=n^{2}
$$

Moreover, we find

$>\operatorname{subs}(t=0, \operatorname{diff}(G(t), t \$ 4))$ :

$>\operatorname{Sum}\left(\sec ^{4}(k * \operatorname{Pi} / n), k=0 . . n-1\right)=\operatorname{factor}(\operatorname{simplify}(\% / 4 !))$;

$$
\sum_{k=0}^{n-1} \sec ^{4}\left(\frac{k \pi}{n}\right)=\frac{n^{2}}{3}\left(n^{2}+2\right)
$$

Here we have used Maple V Release 5, but the computations could also be reproduced with, for example, Mathematica, Mathcad, or any other computer algebra system. The further results obtained by Maple will be listed in the appendix.

CASE 2. Let $n$ be an even positive integer. Deleting the term for $k=n / 2$ from $S_{p}(n)$, we reset

$$
\mathscr{S}_{p}(n)=\sum_{k=0, k \neq n / 2}^{n-1} \sec ^{2 p}\left(\frac{k \pi}{n}\right) .
$$


Similarly, on separating off the term for $k=n / 2$ from (13), we have

$$
1+n \cot n \theta \cot \theta=\sum_{k=0, k \neq n / 2}^{n-1} \frac{-\cos ^{2} \theta}{\cos ^{2} \theta-\cos ^{2}(k \pi / n)} .
$$

In analogy to (14), we find that the generating function of $\mathscr{S}_{p}(n)$ has the form

$$
\mathscr{G}(n, t)=1+n \cot n \theta \cot \theta
$$

where $t=\cos \theta$. Thus, explicitly, we have

$$
\mathscr{G}(n, t)=1-\frac{n t}{\sqrt{1-t^{2}}} \cot (n \arcsin t),
$$

where the fact that $n$ is even and the relation $\arccos \theta=\pi / 2-\arcsin \theta$ have been used. Therefore,

$$
\mathscr{S}_{p}(n)=\sum_{k=0, k \neq n / 2}^{n-1} \sec ^{2 p}\left(\frac{k \pi}{n}\right)=\lim _{t \rightarrow 0} \frac{\varphi^{(2 p)}(n, t)}{(2 p) !} .
$$

As we have done in Case 1, the following Maple commands display

$>$ assume $(n$, integer $)$ :

$>g:=t->1-n * t * \cot (n * \arcsin (t)) / \operatorname{sqr} t\left(1-t^{2}\right):$

$>\operatorname{limit}(\operatorname{diff}(g(t), t \$ 2), t=0)$ :

$>\operatorname{Sum}\left(\sec ^{2}(k * P i / n), k=0 . . n-1\right)=\operatorname{factor}(\operatorname{simplify}(\% / 2 !))$;

$$
\sum_{k=0, k \neq n / 2}^{n-1} \sec ^{2}\left(\frac{k \pi}{n}\right)=\frac{1}{3}\left(n^{2}-1\right),
$$

and

$>\operatorname{limit}(\operatorname{diff}(g(t), t \$ 4), t=0)$ :

$>\operatorname{Sum}\left(\sec ^{4}(k * P i / n), k=0 . . n-1\right)=$ factor $(\operatorname{simplify}(\% / 4 !))$;

$$
\sum_{k=0, k \neq n / 2}^{n-1} \sec ^{4}\left(\frac{k \pi}{n}\right)=\frac{1}{45}\left(n^{2}-1\right)\left(n^{2}+11\right) .
$$

Next, we evaluate the power sums for the cosecant function. Let $p$ and $n$ be positive integers. Set

$$
C_{p}(n)=\sum_{k=1}^{n-1} \csc ^{2 p}\left(\frac{k \pi}{n}\right) .
$$

On separating off the term for $k=0$ from (10), and multiplying the remaining sums by $\tan \theta \sin \theta$, we find

$$
\begin{aligned}
1-n \cot n \theta \tan \theta & =\sum_{k=1}^{n-1} \frac{\sin ^{2} \theta}{\sin ^{2}(k \pi / n)-\sin ^{2} \theta} \\
& =\sum_{k=1}^{n-1} \frac{[\sin \theta / \sin (k \pi / n)]^{2}}{1-[\sin \theta / \sin (k \pi / n)]^{2}}=\sum_{p=1}^{\infty}\left(\sum_{k=1}^{n-1} \csc ^{2 p}\left(\frac{k \pi}{n}\right)\right) \sin ^{2 p} \theta .
\end{aligned}
$$


In analogy to (14), this implies that a generating function of $C_{p}(n)$ has the form

$$
\mathscr{F}(n, t)=1-n \cot n \theta \tan \theta,
$$

where $t=\sin \theta$. Explicitly, we have

$$
\mathscr{F}(n, t)=1-\frac{n t}{\sqrt{1-t^{2}}} \cot (n \arcsin t)
$$

Moreover, we find

$$
C_{p}(n)=\mathscr{F}^{(2 p)}(n, 0) /(2 p) !=\mathscr{S}_{p}(n)
$$

Therefore, in view of (25) and (26), we find

$$
\begin{aligned}
& \sum_{k=1}^{n-1} \csc ^{2}\left(\frac{k \pi}{n}\right)=\sum_{k=0, k \neq n / 2}^{n-1} \sec ^{2}\left(\frac{k \pi}{n}\right)=\frac{1}{3}\left(n^{2}-1\right) \\
& \sum_{k=1}^{n-1} \csc ^{4}\left(\frac{k \pi}{n}\right)=\sum_{k=0, k \neq n / 2}^{n-1} \sec ^{4}\left(\frac{k \pi}{n}\right)=\frac{1}{45}\left(n^{2}-1\right)\left(n^{2}+11\right) .
\end{aligned}
$$

Finally, using the trigonometric identity $\cot ^{2} \theta=\csc ^{2} \theta-1$, we transform the above identities as follows:

$$
\begin{aligned}
& \sum_{k=1}^{n-1} \cot ^{2}\left(\frac{k \pi}{n}\right)=\frac{(n-1)(n-2)}{3}, \\
& \sum_{k=1}^{n-1} \cot ^{4}\left(\frac{k \pi}{n}\right)=\frac{(n-1)(n-2)}{45}\left(n^{2}+3 n-13\right) .
\end{aligned}
$$

Using the trigonometric identity $\tan ^{2} \theta=\sec ^{2} \theta-1$ in (17) and (19) yields

$$
\begin{aligned}
& \sum_{k=1}^{n-1} \tan ^{2}\left(\frac{k \pi}{n}\right)=n(n-1) \\
& \sum_{k=1}^{n-1} \tan ^{4}\left(\frac{k \pi}{n}\right)=\frac{n(n-1)}{3}\left(n^{2}+n-3\right)
\end{aligned}
$$

in (25) and (26) the transformation produces

$$
\begin{aligned}
& \sum_{k=1, k \neq n / 2}^{n-1} \tan ^{2}\left(\frac{k \pi}{n}\right)=\frac{(n-1)(n-2)}{3}, \\
& \sum_{k=1, k \neq n / 2}^{n-1} \tan ^{4}\left(\frac{k \pi}{n}\right)=\frac{(n-1)(n-2)}{45}\left(n^{2}+3 n-13\right) .
\end{aligned}
$$

We have established a variety of power sum identities. It is interesting to see how Maple appeared in the derivations. For completeness, we provide additional power sum identities, which are produced by Maple, in the appendix. 


\section{Appendix}

A. A table of more power sums

A.1. Power sum of secant. When $n$ is odd

$$
\begin{aligned}
& \sum_{k=0}^{n-1} \sec ^{6}\left(\frac{k \pi}{n}\right)=\frac{n^{2}}{15}\left(2 n^{4}+5 n^{2}+8\right), \\
& \sum_{k=0}^{n-1} \sec ^{8}\left(\frac{k \pi}{n}\right)=\frac{n^{2}}{315}\left(17 n^{6}+56 n^{4}+98 n^{2}+144\right), \\
& \sum_{k=0}^{n-1} \sec ^{10}\left(\frac{k \pi}{n}\right)=\frac{n^{2}}{2835}\left(62 n^{8}+255 n^{6}+546 n^{4}+820 n^{2}+1152\right) .
\end{aligned}
$$

When $n$ is even

$$
\begin{aligned}
\sum_{k=0, k \neq n / 2}^{n-1} \sec ^{6}\left(\frac{k \pi}{n}\right) & =\frac{\left(n^{2}-1\right)}{945}\left(2 n^{4}+23 n^{2}+191\right), \\
\sum_{k=0, k \neq n / 2}^{n-1} \sec ^{8}\left(\frac{k \pi}{n}\right) & =\frac{\left(n^{2}-1\right)}{14175}\left(3 n^{6}+43 n^{4}+337 n^{2}+2497\right), \\
\sum_{k=0, k \neq n / 2}^{n-1} \sec ^{10}\left(\frac{k \pi}{n}\right) & =\frac{\left(n^{2}-1\right)}{93555}\left(2 n^{8}+35 n^{6}+321 n^{4}+2125 n^{2}+14797\right) .
\end{aligned}
$$

\section{A.2. Power sum of cosecant.}

$$
\begin{aligned}
& \sum_{k=1}^{n-1} \csc ^{6}\left(\frac{k \pi}{n}\right)=\frac{\left(n^{2}-1\right)}{945}\left(2 n^{4}+23 n^{2}+191\right) \\
& \sum_{k=1}^{n-1} \csc ^{8}\left(\frac{k \pi}{n}\right)=\frac{\left(n^{2}-1\right)}{14175}\left(3 n^{6}+43 n^{4}+337 n^{2}+2497\right), \\
& \sum_{k=1}^{n-1} \csc ^{10}\left(\frac{k \pi}{n}\right)=\frac{\left(n^{2}-1\right)}{93555}\left(2 n^{8}+35 n^{6}+321 n^{4}+2125 n^{2}+14797\right)
\end{aligned}
$$

A.3. Power sum of tangent. When $n$ is odd

$$
\begin{aligned}
\sum_{k=1}^{n-1} \tan ^{6}\left(\frac{k \pi}{n}\right) & =\frac{n(n-1)}{15}\left(2 n^{4}+2 n^{3}-8 n^{2}-8 n+15\right) \\
\sum_{k=1}^{n-1} \tan ^{8}\left(\frac{k \pi}{n}\right) & =\frac{n(n-1)}{315}\left(17 n^{6}+17 n^{5}-95 n^{4}-95 n^{3}+213 n^{2}+213 n-315\right) \\
\sum_{k=1}^{n-1} \tan ^{10}\left(\frac{k \pi}{n}\right) & =\frac{n(n-1)}{2385}\left(62 n^{8}+62 n^{7}-448 n^{6}-48 n^{5}+1358 n^{4}\right. \\
& \left.+1358 n^{3}-2232 n^{2}-2232 n+2835\right)
\end{aligned}
$$


When $n$ is even

$$
\begin{aligned}
& \sum_{k=1, k \neq n / 2}^{n-1} \tan ^{6}\left(\frac{k \pi}{n}\right)=\frac{(n-1)(n-2)}{945}\left(2 n^{4}+6 n^{3}-28 n^{2}-96 n+251\right) \\
& \begin{aligned}
& \sum_{k=1, k \neq n / 2}^{n-1} \tan ^{8}\left(\frac{k \pi}{n}\right)=\frac{(n-1)(n-2)}{14175}\left(3 n^{6}+9 n^{5}-59 n^{4}-195 n^{3}\right. \\
&+\left.457 n^{2}+1761 n-3551\right) \\
& \sum_{k=1, k \neq n / 2}^{n-1} \tan ^{10}\left(\frac{k \pi}{n}\right)=\frac{(n-1)(n-2)}{93555}\left(2 n^{8}+6 n^{7}-52 n^{6}-168 n^{5}+546 n^{4}\right. \\
&\left.+1974 n^{3}-3068 n^{2}-13152 n+22417\right)
\end{aligned}
\end{aligned}
$$

\section{A.4. Power sum of cotangent.}

$$
\begin{aligned}
\sum_{k=1}^{n-1} \cot ^{6}\left(\frac{k \pi}{n}\right)=\frac{(n-1)(n-2)}{945}( & \left.2 n^{4}+6 n^{3}-28 n^{2}-96 n+251\right) \\
\sum_{k=1}^{n-1} \cot ^{8}\left(\frac{k \pi}{n}\right)=\frac{(n-1)(n-2)}{14175}( & 3 n^{6}+9 n^{5}-59 n^{4}-195 n^{3} \\
& \left.+475 n^{2}+1761 n-3551\right) \\
\sum_{k=1}^{n-1} \cot ^{10}\left(\frac{k \pi}{n}\right)=\frac{(n-1)(n-2)}{93555}( & 2 n^{8}+6 n^{7}-52 n^{6}-168 n^{5}+546 n^{4} \\
& \left.+1974 n^{3}-3068 n^{2}-13152 n+22417\right)
\end{aligned}
$$

\section{REFERENCES}

[1] H. Chen, On a new trigonometric identity, to appear in Internat. J. Math. Ed. Sci. Tech.

[2] E. R. Hansen, A Table of Series and Products, Prentice Hall, New Jersey, 1975.

[3] T. J. Rivlin, Chebyshev Polynomials, 2nd ed., John Wiley, New York, 1990.

[4] K. R. Stromberg, Introduction to Classical Real Analysis, Wadsworth, California, 1981.

Hongwei Chen: Department of Mathematics, Christopher Newport University, NEWPORT NEWS, VA 23606, USA

E-mail address: hchen@pcs. cnu . edu 


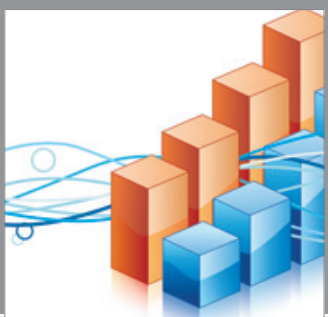

Advances in

Operations Research

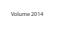

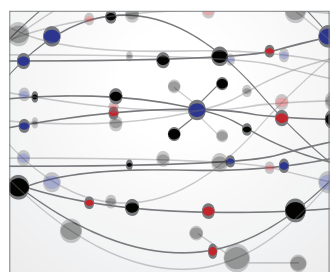

\section{The Scientific} World Journal
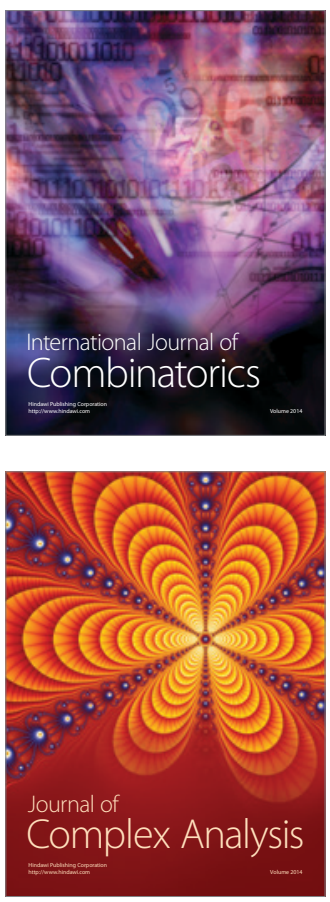

International Journal of

Mathematics and

Mathematical

Sciences
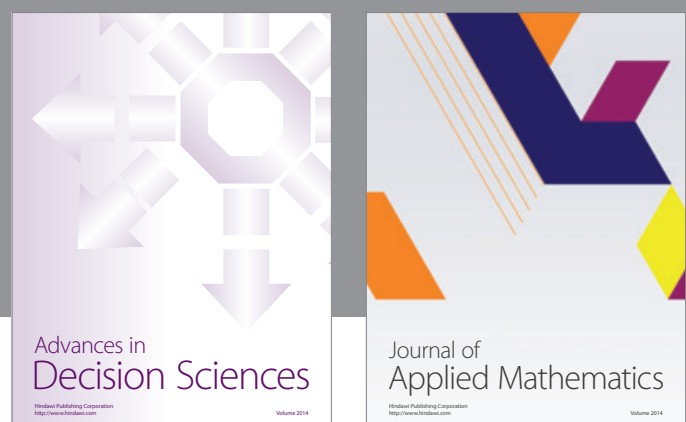

Journal of

Applied Mathematics
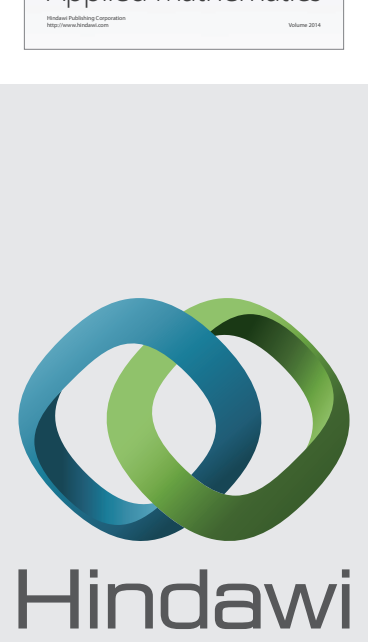

Submit your manuscripts at http://www.hindawi.com
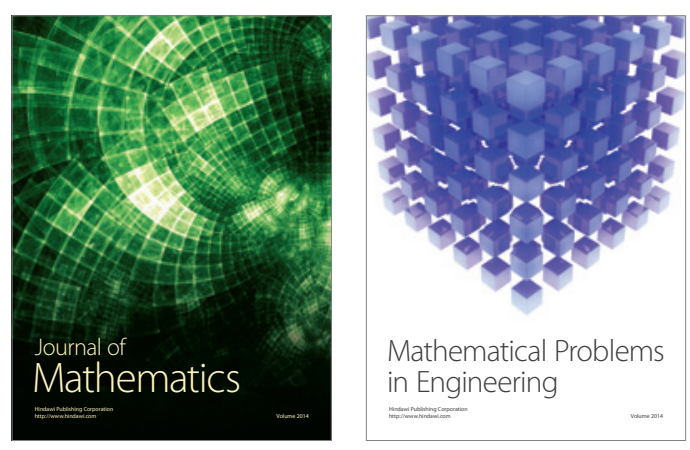

Mathematical Problems in Engineering
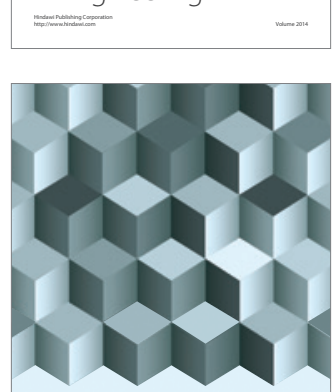

Journal of

Function Spaces
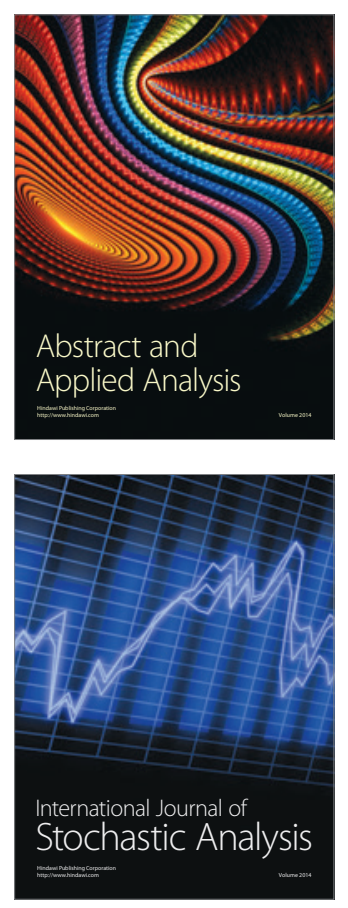

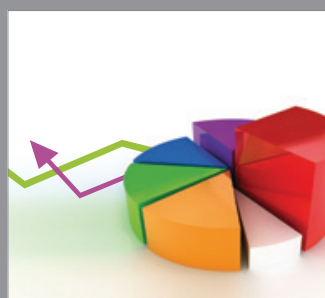

ournal of

Probability and Statistics

Promensencen
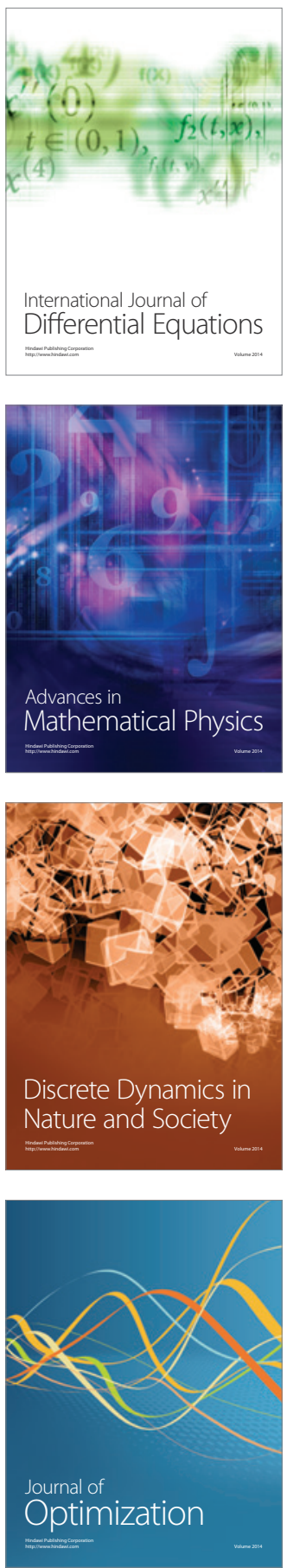